\title{
PROXIMATE ORDER (R) OF ENTIRE FUNCTIONS REPRESENTED BY DIRICHLET SERIES (IV)

\author{
by PAWAN KUMAR KAMTHAN
}

(Received 23 July, 1963)

1. Let

$$
f(s)=\sum_{n=1}^{\infty} a_{n} e^{s \lambda_{n}} \quad(s=\sigma+i t),
$$

where $0=\lambda_{0}<\lambda_{1}<\ldots, \lambda_{n} \rightarrow \infty$ as $n \rightarrow \infty$, and $\lim \inf \left(\lambda_{n+1}-\lambda_{n}\right)=h>0$, be an entire function represented by a Dirichlet series whose order (R) and proximate order (R) are respectively $\rho(0<\rho<\infty)$ and $\rho(\sigma)$. For proximate order (R) and its properties, see the paper of Balaguer [4, p. 28].

In $\$ 2$ I prove a general theorem involving proximate order $(R)$, and in $\$ 3 I$ apply it to give an important application in the theory of entire functions represented by Dirichlet series. $\S 4$ is exclusively devoted to illustrating the best possible nature of this application.

2. Define

$$
A(\sigma)=\exp \left(\int_{0}^{\sigma} \rho(t) d t\right), \quad B(\sigma)=\int_{0}^{\sigma} n(t) \rho(t) d t,
$$

where $n(t)$ is a non-decreasing function of $t$, at least for $t \geqq t_{0}$, and is continuous almost everywhere in $\left(t_{0}, t\right)$. Let

$$
\begin{aligned}
& \lim _{\sigma \rightarrow \infty} \sup \{n(\sigma) / A(\sigma)\}=C, \quad \lim _{\sigma \rightarrow \infty} \inf \{n(\sigma) / A(\sigma)\}=D, \\
& \lim _{\sigma \rightarrow \infty} \sup \{B(\sigma) / A(\sigma)\}=E, \quad \lim _{\sigma \rightarrow \infty} \inf \{B(\sigma) / A(\sigma)\}=F .
\end{aligned}
$$

I have elsewhere shown $[1,(4.1)]$ that

$$
e E \geqq C e^{D / C} \geqq C .
$$

THEOREM. Let $0<\rho<\infty$ and $0<E<\infty$; then

$$
1 \leqq \lim _{\sigma \rightarrow \infty} \sup \frac{B^{\prime}(\sigma)}{E \rho(\rho) A(\sigma)} \leqq \frac{C}{E} \leqq e,
$$

where $\sigma \rightarrow \infty$ excluding a set of measure zero and $B^{\prime}(\sigma)$ is the first derivative of $B(\sigma)$.

Proof. We have, for almost all $\sigma \geqq \sigma_{0}$,

$$
\begin{aligned}
B^{\prime}(\sigma) & =n(\sigma) \rho(\sigma)<(C+\varepsilon) A(\sigma) \rho(\sigma) \\
& \leqq(e E+\varepsilon) A(\sigma) \rho(\sigma),
\end{aligned}
$$

by (2.1), where $\varepsilon$ is an arbitrarily small positive number. Hence

$$
\lim _{\sigma \rightarrow \infty} \sup \frac{B^{\prime}(\sigma)}{E A(\sigma) \rho(\sigma)} \leqq \frac{C}{E} \leqq e .
$$

$\dagger$ From now on it will be assumed that $\sigma \rightarrow \infty$ excluding a set of measure zero. 
Next, suppose that the left-hand inequality in (2.2) is false; then if $\delta<1$, we have

$$
\frac{B^{\prime}(\sigma)}{E A(\sigma) \rho(\sigma)}<\delta_{1} \quad\left(\delta<\delta_{1}<1, \sigma \geqq \sigma_{0}\right) .
$$

Then

and consequently

$$
\begin{aligned}
B(\sigma)-B\left(\sigma_{0}\right) & =\int_{\sigma_{0}}^{\sigma} B^{\prime}(x) d x<E \delta_{1} \int_{\sigma_{0}}^{\sigma} A(x) \rho(x) d x \\
& =E \delta_{1} \int_{\sigma_{0}}^{\sigma} A^{\prime}(x) d x
\end{aligned}
$$

$$
\lim _{\sigma \rightarrow \infty} \sup \frac{B(\sigma)}{A(\sigma)} \leqq E \delta_{1}<E,
$$

which is inconsistent with the fact that

$$
\lim _{\sigma \rightarrow \infty} \sup \frac{B(\sigma)}{A(\sigma)}=E ;
$$

the result follows.

3. An application. Let $\rho(\sigma)=\rho, B(\sigma)=\rho \log \mu(\sigma)$ and $n(\sigma)=\lambda_{\nu(\sigma)}$, where $\mu(\sigma)$ and $\lambda_{v(\sigma)}$ are respectively the maximum term of $f(s)$ and the rank of the maximum term. Then we have from the above theorem:

$$
1 \leqq \lim _{\sigma \rightarrow \infty} \sup \frac{\mu^{\prime}(\sigma)}{\rho T \mu(\sigma) e^{\rho \sigma}} \leqq e,
$$

where $\mu^{\prime}(\sigma)$ is the first derivative of $\mu(\sigma)$ and

$$
T=\lim _{\sigma \rightarrow \infty} \sup \frac{\log \mu(\sigma)}{e^{\rho \sigma}}(0<T<\infty),
$$

$T$ being the type of $f(s)$. The result (3.1) has been obtained earlier by the author [2].

4. First, I construct an example which shows that the lower bound in (3.1) cannot be greater than 1 .

Example 1. Let $0<T<\infty, 0<\rho<\infty$, and

$$
f(s)=\sum_{n=1}^{\infty} \frac{T^{n} e^{[n \rho] s}}{n !}
$$

Suppose now that $\chi_{n}$ denotes the rectified ratio in $f(s)$. Then

$$
\chi_{n}=\log \left(\frac{n}{T}\right) /([n \rho]-[(n-1) \rho])
$$


Hence, for $\chi_{n} \leqq \sigma<\chi_{n+1}$, we have

$$
\mu(\sigma)=\frac{T^{n} e^{[n \rho] \sigma}}{n !}, \quad \mu^{\prime}(\sigma)=\frac{[n \rho] T^{n} e^{[n \rho] \sigma}}{n !} .
$$

Now obviously $f(s)$ is an entire function of order (R) $\rho$. By (4.1),

$$
\frac{\log \mu(\sigma)}{e^{\sigma \rho}}=\frac{[n \rho] \sigma+n \log T-\log n !}{e^{\sigma \rho}},
$$

and so, for large $n$,

$$
\frac{\log \mu\left(\chi_{n}\right)}{e^{\rho \chi_{n}}} \sim \frac{n \log (n / T)+n \log T-n \log n+n-\frac{1}{2} \log n}{e^{\log (n / T)}} \sim T .
$$

Since

$$
\frac{\log \mu\left(\chi_{n+1}\right)}{e^{\rho \chi_{n}+1}} \leqq \frac{\log \mu(\sigma)}{e^{\rho \sigma}} \leqq \frac{\log \mu\left(\chi_{n}\right)}{e^{\rho \chi_{n}}}
$$

it follows that

$$
\lim _{\sigma \rightarrow \infty} \sup \frac{\log \mu(\sigma)}{e^{\rho \sigma}}=T
$$

and hence $f(s)$ is of type $T$. Further, by (4.1),

$$
\frac{\mu^{\prime}\left(\chi_{n}\right)}{\rho T \mu\left(\chi_{n}\right) e^{\rho \chi_{n}}} \rightarrow 1 \quad \text { as } n \rightarrow \infty,
$$

and it follows similarly that

$$
\lim _{\sigma \rightarrow \infty} \inf \frac{\mu^{\prime}(\sigma)}{\rho T \mu(\sigma) e^{\rho \sigma}}=1 .
$$

Next, consider the following example which shows that the upper bound $e$ in (3.1) cannot be diminished. Srivastava [3] also makes use of it for another purpose.

Example 2. Let

$$
f(s)=\sum_{n=1}^{\infty}\left\{\frac{e^{s}}{\mu_{n}}\right\}^{e \rho T \mu_{n}^{\rho}},
$$

where

$$
\mu_{1}=1, P=1+[\rho], \quad \mu_{n+1}=\exp \left(\mu_{n}^{P}\right) \text { for } n=1,2, \ldots
$$

Srivastava [3, p. 144] has shown that $f(s)$ is an entire function of $\operatorname{order}(\mathrm{R}) \rho$ and type $T$. Also

$$
\mu(\sigma)=\left\{\frac{e^{\sigma}}{\mu_{n}}\right\}^{e \rho T \mu_{n}^{\rho}},
$$

B 


$$
\phi(n) \leqq \sigma<\phi(n+1),
$$

where

$$
\phi(n)=\frac{1}{\mu_{n}^{\rho}-\mu_{n-1}^{\rho}}\left\{\mu_{n}^{\rho} \log \mu_{n}-\mu_{n-1}^{\rho} \log \mu_{n-1}\right\} .
$$

Now it is obvious that for large $n$, say $n>n_{0}$, we have

$$
\log \mu_{n}<\phi(n)<\log \left(\mu_{n}+\mu_{n}^{\kappa}\right)<\log \mu_{n}+1 / \rho<\log \mu_{n+1}<\phi(n+1) \quad \text { if } 0<\kappa<1 .
$$

Again, for the above function,

$$
\mu^{\prime}(\sigma)=e \rho T \mu_{n}^{\rho}\left\{\frac{e^{\sigma}}{\mu_{n}}\right\}^{e \rho T \mu_{n}^{\rho}},
$$

where $\sigma$ lies in the interval defined by (4.3). Therefore, from (4.2) and (4.4), we get

$$
-\frac{\mu^{\prime}(\sigma)}{\rho T \mu(\sigma) e^{\rho \sigma}}=\frac{e \mu_{n}^{\rho}}{e^{\rho \sigma}}
$$

Let $\sigma=\log \left(\mu_{n}+\mu_{n}^{k}\right)=\delta_{n}$. Then, from (4.5), we obtain

$$
\frac{\mu^{\prime}\left(\delta_{n}\right)}{\rho T \mu\left(\delta_{n}\right) e^{\rho \delta_{n}}}=\frac{e \mu_{n}^{\rho}}{\left(\mu_{n}+\mu_{n}^{\kappa}\right)^{\rho}} \rightarrow e \quad \text { as } n \rightarrow \infty .
$$

Therefore

$$
\lim _{\sigma \rightarrow \infty} \sup \frac{\mu^{\prime}(\sigma)}{\rho T \mu(\sigma) e^{\rho \sigma}} \geqq e
$$

and this, when combined with (3.1), shows that for this function

$$
\lim _{\sigma \rightarrow \infty} \sup \frac{\mu^{\prime}(\sigma)}{\rho T \mu(\sigma) e^{\rho \sigma}}=e .
$$

\section{REFERENCES}

1. P. K. Kamthan, Proximate order (R) of entire functions represented by Dirichlet series, (II), Collectanea Math. 15 (1963), 71-75.

2. P. K. Kamthan, On the order and type of entire Dirichlet series, Math. Student (in Press).

3. K. N. Srivastava, On the maximum term of an entire Dirichlet series, Proc. Nat. Acad. Sci. India, 27 (1958), 134-146.

4. F. Sunyer i Balaguer, Sobra la distribución de los valores de una función entera representada por una serie de Dirichlet lagunar, Rev. Acad. Ci. Madrid (2) 5 (1950), 25-73.

UNIVERSITY OF DELHI, DELHI 6 INDIA 